\title{
MIND TRANSITION MODEL -A UNIFIED MODEL OF AHP AND ANP-
}

\author{
Keikichi OHSAWA, Chikako MIYAKE and Masaaki SHINOHARA \\ Department of Mathematical Information Engineering \\ College of Industrial Technology, Nihon University \\ 1-2-1 Izumi-chou, Narashino, Chiba 275-8575, Japan \\ m7sinoha@cit.nihon-u.ac.jp
}

Keywords: unified model of AHP and ANP, decision maker's mind transition, decision making, mental state psychology

Summary: Mind Transition Model (MTM), which is a unified model of AHP and ANP, is proposed. MTM is a Markov chain network representing one's mental state transition. Both AHP and ANP can be interpreted as MTM networks.

\begin{abstract}
A unified treatment of AHP and ANP is presented in this paper. We propose MTM, or Mind Transition Model, which is a unified model of AHP and ANP. MTM is a Markov chain network with its states corresponding to one's mind states and its transition arcs corresponding to one's mind transitions. MTM enables one simulate one's mind transition profile, thus with MTM we can simulate the mind transition profiles when using AHP and ANP. Both in AHP and ANP the priority weight of an element corresponds to the stationary state probability of the element. The bigger is the stationary state probability of an element, the higher is the priority weight of the element. The priority weight of an element is interpreted being proportional to the time duration of the mind staying in the state for the element.
\end{abstract}

Table 1 Comparison of AHP and ANP

\begin{tabular}{c|c|c}
\hline Structure & AHP & ANP \\
\hline $\begin{array}{c}\text { Local pri ori ty wei ght det ernnati on } \\
\text { A ternati ve pri ori ty wei ght } \\
\text { deternnati on }\end{array}$ & Pai red compari son, etc & Pai red conpari son, etc \\
\hline Markov chai $n$ & Non-ergodi c(or transi ent) & Ergodi c and non- ergodi c \\
\hline
\end{tabular}




\section{Mind Transition Model (MTM)}

Mind Transition Model, or MTM, is a Markov chain network whose nodes correspond to mind states and arcs correspond to mind transitions. When one thinks of something, we can say that his/her mind is in the state of the something. At the next moment, he/she may think of another thing. Then, his/her mind state may change to the other thing. How long one's mind stays at a state or at a group of states reflects how frequently he/she thinks of the element associated with of the state or the concept associated with the group of states. So the stationary state probability of the state for an element can be, in a sense, interpreted as the priority weight of the el ement. It is questionable whether or not the priority weight is linearly proportional to the stationary state probability. But we assume they are linearly proportional to each other.

\section{Nonstrongly-connected 3-level ANP interpreted as MTM}

We consider a nonstrongly-connected 3-level ANP, or Saaty's car-buying example with outer dependence and goal level. The 3-level ANP is obtained either by adding to the bipartite ANP the goal "Car Choice" and priority weight arcs from the goal to each of the criteria or by adding to the AHP priority weight arcs from each of the alternatives to each of the criteria. Since there is no priority weight arcs from the criterion to the goal, the 3-level ANP network is not strongly-connected, and hence the corresponding MTM as it is, is non-ergodic and the goal is a transient state with its stationary state probability being zero. Therefore, we need to modify the original 3-level non-ergodic ANP network to form an ergodic MTM network. Among the many ways of transforming non-ergodic networks into ergodic networks, a method of adding the absent-mind state will be presented next.

The ergodic Markov chain MTM is constructed by adding a new state, called "absent-mind state", to the original 3-level non-ergodic ANP network. An arc with transition probability $p$ is assigned to the absent-mind state from each of criterion states and alternative states. This probability $p$ is call volatility rate.

Remaining transition probabilities are normalized so as to satisfy the probability condition, or that the sum of transition probabilities from a state is unity. From the

absent-mind state to the goal state there assigned an arc with transition probability $=1$. The resultant Markov chain is ergodic, and moreover, regular (not periodic).

While one is thinking over the car-choice problem, at some time one may think of a criterion and at next moment one may think of an alternative. But with certain probability, or volatility rate $p$, one may stop thinking over the car-choice problem and think of another thing. This transition out of the present-interesting is modeled by the transition to the absent-mind state. After a while staying in the absent-mind state, one may again start thinking over the car-choice problem, which is represented by the transition from the absent-mind state to the goal state. 